\title{
Endovascular repair of acute type B thoracic aortic dissection
}

\author{
Alice Le Huu', Ourania Preventza ${ }^{1,2}$ \\ ${ }^{1}$ Division of Cardiothoracic Surgery, Michael E. DeBakey Department of Surgery, Baylor College of Medicine, Houston, Texas, USA; ${ }^{2}$ Section of \\ Adult Cardiac Surgery, Department of Cardiovascular Surgery, Texas Heart Institute, Houston, Texas, USA \\ Correspondence to: Ourania Preventza, MD. Division of Cardiothoracic Surgery, Michael E. DeBakey Department of Surgery, Baylor College of \\ Medicine, BCM 390, One Baylor Plaza, Houston, Texas 77030, USA. Email: preventz@bcm.edu.
}

\begin{abstract}
Approximately one-third of patients with acute Stanford type B or DeBakey type III aortic dissection (TBAD) will develop complications, including persistent symptoms, malperfusion, enlarging aneurysms and impending rupture. In these cases, TBAD becomes a surgical emergency that requires endovascular intervention to complement the medical therapy. The immediate goal of endovascular therapy is to reestablish flow to the true lumen, stabilize the aneurysm and prevent rupture. Long-term goals are the remodeling of the descending thoracic aorta and the prevention of further surgeries in the thoracoabdominal aorta. In this report, we describe our step-by-step endovascular approach to TBAD repair.
\end{abstract}

Keywords: Aneurysm; dissecting; aorta; thoracic; endovascular procedures; stents grafts; vascular grafting

Submitted Sep 22, 2021. Accepted for publication Oct 24, 2021.

doi: 10.21037/acs-2021-taes-26

View this article at: https://dx.doi.org/10.21037/acs-2021-taes-26

\section{Introduction}

Acute Stanford type B or DeBakey type III aortic dissection (TBAD) originating distal to the left subclavian artery is a medical condition that is typically treated with anti-impulse therapy. However, as many as $30 \%$ of patients with this type of dissection will develop complications, including persistent symptoms, malperfusion, enlarging aneurysms and impending rupture (1-3). In these cases, TBAD becomes a surgical emergency that requires endovascular intervention to complement the medical therapy. Thoracic endovascular aortic repair (TEVAR) is an approach that can immediately reestablish flow to the true lumen, stabilize the aneurysm and prevent rupture, while lowering the mortality rate to approximately $14 \%$. Long-term benefits of TEVAR include the remodeling of the descending thoracic aorta and elimination of subsequent procedures in the thoracoabdominal aorta.

TEVAR has long been considered a viable therapeutic option for complicated TBAD; however, its ability to promote thrombosis of the false lumen and to prevent progression of the aneurysm $(4,5)$ indicate that it is also appropriate for uncomplicated scenarios. As a result, there has been a subtle paradigm shift in the considerations for TEVAR use (6). Originally used to treat descending thoracic aortic aneurysm disease, TEVAR grafts are now approved by the US Food and Drug Administration for the treatment of acute TBAD, which has increased interest in endovascular technology and widened the spectrum of patients for which it is applicable.

In the following section, we describe our typical TEVAR operative technique for TBAD repair, including principles and helpful tips based on our experience with this procedure.

\section{Operative technique}

\section{Preparation}

In terms of patient-specific surgical planning, detailed examination of the preoperative imaging-either contrastenhanced computed tomography (CT) or noncontrast magnetic resonance imaging - is essential. We prefer to use a gated contrast-enhanced CT scan, if possible. Of interest are adequate access (angulation, caliber and calcification of iliac vessels), the quality of the aorta, the tortuosity of the aortic arch, the presence of calcifications or atherosclerotic plaques and the caliber of the ascending aorta; this 


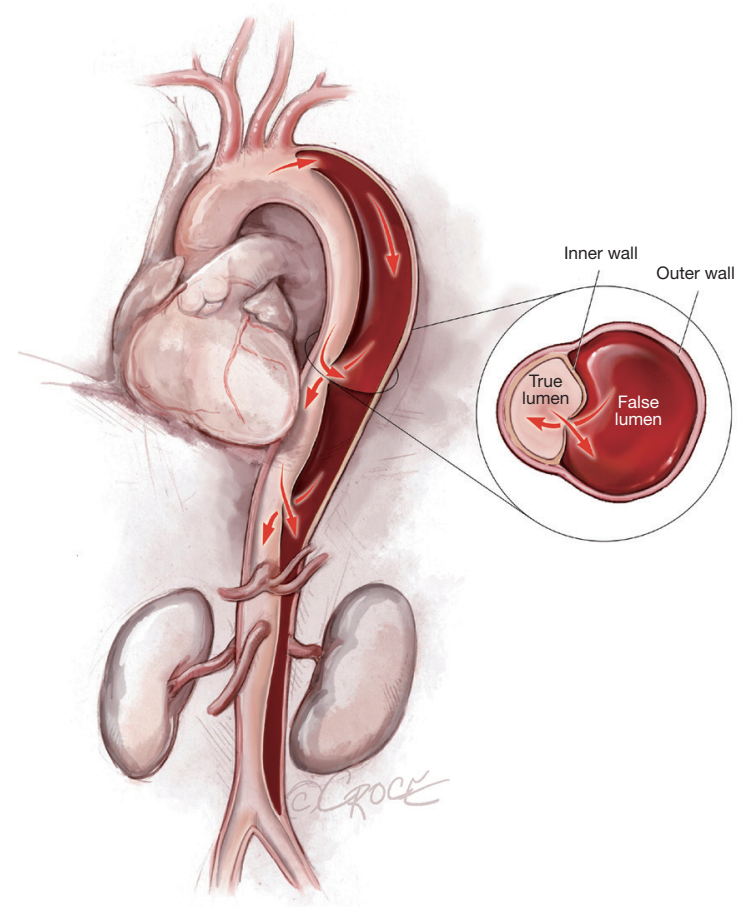

Figure 1 Acute Stanford type B aortic dissection extending from the level of the left subclavian artery to the aortic bifurcation.

information is vital for any thoracic aortic endovascular case. In cases of aortic dissection, it is particularly important to know the involvement of the access vessels in the dissection process, the location of the aortic dissection entry tear and its proximity to the aortic arch, and the relationship of the origin of the visceral vessels to the false and the true lumen of the thoracoabdominal aorta. Of note, acute TBAD is a dynamic process, and initial imaging obtained at the time of the patient's admission to the hospital may need to be updated, as the patient's condition may have changed over the course of 48-72 hours of disease progression.

The diameter and length of the stent graft are determined on the basis of preoperative CT scans, intravascular ultrasound (IVUS) imaging, and aortograms. Whereas device oversizing of $15-25 \%$ can be acceptable for aortic aneurysm repair, $<10 \%$ oversizing is advisable, and $0 \%$ (no oversizing) is preferable, for acute dissection repair. Oversizing the stent graft by $>10 \%$ can result in retrograde proximal aortic dissection and potential folding of the graft.

\section{Exposition}

We acknowledge that there are various ways to perform
TEVAR for acute TBAD (Figure 1). Whereas TEVAR can be performed under regional or local anesthesia, our preference is to use general anesthesia. Before positioning the patient, we check and record bilateral pedal pulses by palpation or Doppler ultrasound. We then place the patient in a supine position; self-adhesive medical tape can be used to retract any excess tissue or pannus that could obscure the groins before they and the abdomen are prepped and draped.

Occasionally, femoral access to the true lumen of the thoracoabdominal aorta is difficult, and right radial or right brachial arterial access may become necessary; if radial or brachial access is required, we perform it before we drape the patient. We then place a pulse oximeter on the left hand, preferentially; occasionally, part of the left subclavian artery may need to be covered by the graft to prevent proximal endoleak, in which case ensuring adequate pulse oximeter saturation will be important.

\section{Operation}

In this section, we describe our current process for TBAD repair. We have found this approach to be a safe and reproducible way to achieve highly successful results.

\section{Establishing femoral access}

In the past, we performed open surgical exposure of the femoral vessels for any sheath size $>9$ F. However, given the evolution of perclosure devices and techniques, we now access the femoral vessels percutaneously regardless of sheath size-unless the patient has very calcified femoral vessels, in which case open surgical exposure is preferable.

We use a Micropuncture Access Set (Cook Medical, Bloomington, IN, USA) under ultrasound guidance to perform the initial puncture (which should be positioned above the femoral bifurcation), after which a $0.018 \mathrm{~mm}$ nitinol wire is threaded through the needle, and a $4 \mathrm{~F}$ catheter is inserted over the wire. Fluoroscopic angiography or just fluoroscopic imaging can be used to assess the location of the wire in relation to the femoral head and to confirm positioning. To circumvent potential dissection or rupture of the iliofemoral vessels, we avoid inserting a large-bore sheath over a soft wire.

Once the appropriate positioning has been confirmed, we replace the $4 \mathrm{~F}$ sheath with a $6 \mathrm{~F}$ sheath and prepare the access site for percutaneous closure, for which we use the Perclose ProGlide ${ }^{\mathrm{TM}}$ SMC System (Abbott). We then dose for heparin, with a target activated clotting time of $260-300$ seconds. 


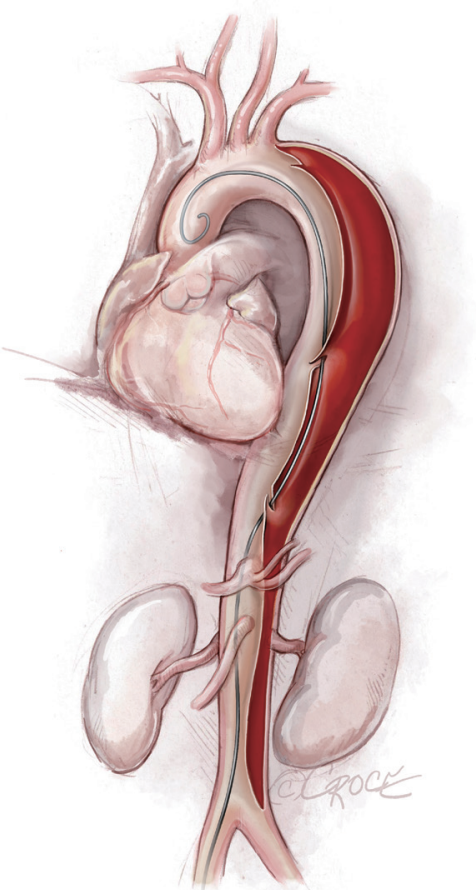

Figure 2 Guidewire placement. In this illustration, the guidewire is placed correctly inside the true lumen throughout the entire length of the thoracoabdominal aorta.

\section{Placing the stent graft}

To access the thoracoabdominal aorta, the arch and the ascending aorta, we use a soft guidewire $(0.035 \times 450 \mathrm{~cm}$ hydrophilic guidewire) followed by an angled $5 \mathrm{~F}$ soft catheter. Ideally, the guidewire will be situated inside the true lumen throughout the length of the thoracoabdominal aorta (Figure 2); however, it is important to realize that the wire can pass between the true lumen and the false lumen through any of the multiple fenestrations between the two lumens (Figure 3). Easy advance of the wire in the ascending aorta confirms that at least the proximal part of the wire is in the true lumen, whereas coiling of the wire, especially at the level of the left subclavian artery, may indicate that the wire is in the false lumen and needs to be redirected to the true lumen. Careful manipulation of the wire (and catheter) is imperative to avoid retrograde ascending aortic dissection; for this reason, we use a combination of IVUS imaging and fluoroscopy to ensure that the wire and catheter are in the true lumen, at least in the areas we plan to use as proximal and distal landing zones (Figures 2,3). We sometimes use an IVUS catheter in lieu of the angled $5 \mathrm{~F}$ soft catheter to access the ascending and thoracoabdominal aortas [our

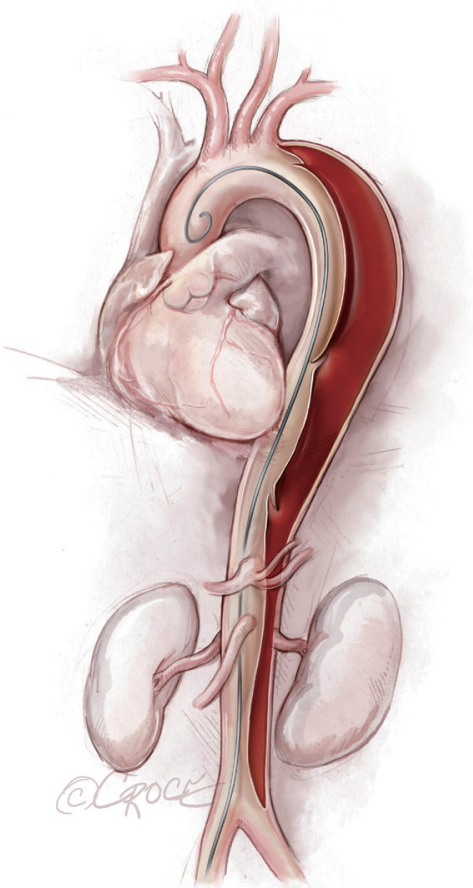

Figure 3 Guidewire placement. During advancement of the soft guidewire, the guidewire can pass in and out of the true lumen. In this illustration, the guidewire is located inside the true lumen initially, then crosses into the false lumen and then back into the true lumen in the ascending aorta.

preference is a Vision PV $8.2 \mathrm{~F} \times 90 \mathrm{~cm}, 8-12 \mathrm{MHz}$ IVUS catheter (Volcano Corporation, San Diego, CA, USA)].

Once we have advanced the wire into the ascending aorta, we use the IVUS catheter to mark the head vessels and the visceral vessels by using a pull-through technique. If angiography is needed, we use a $5 \mathrm{~F} \times 90 \mathrm{~cm}$ Royal Flush Plus pigtail catheter (Cook Medical) to visualize the head vessels and visceral vessels. We can use either the IVUS or the pigtail catheter to confirm the length of the stent graft (we prefer to use the IVUS over a stiff wire instead of a soft wire). If only classic (2-dimensional) imaging is available through the imaging intensifier, after the head vessels and visceral vessels are marked on the display screen, the operating table is locked. The hydrophilic guidewire is exchanged through the IVUS catheter (or soft catheter or pigtail catheter) for a Lunderquist Extra-Stiff $0.035 \times$ $260 \mathrm{~cm}$ tetrafluoroethylene-coated simple or double curve guidewire (Cook Medical).

If we are deploying a stent graft that requires an introducer sheath, we insert a same-size GORE DrySeal 


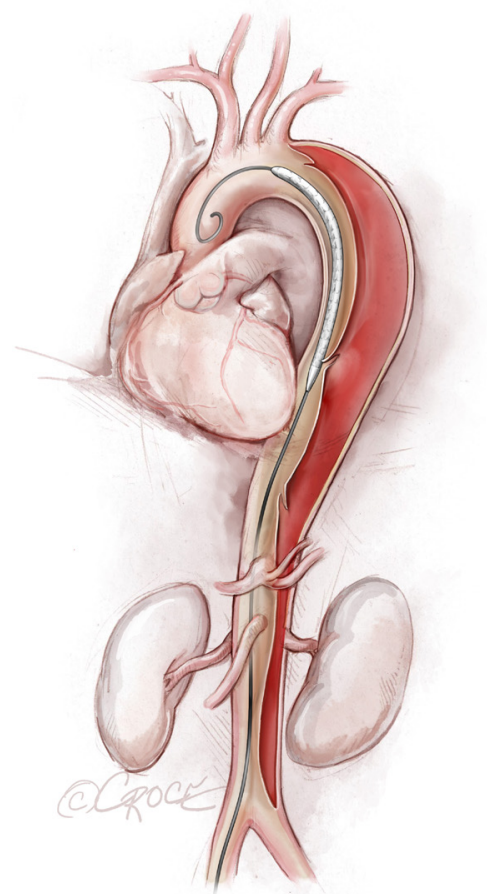

Figure 4 The undeployed stent graft in place. The stent graft device is advanced over an extra stiff guidewire into the thoracoabdominal aorta and is positioned into place. The endovascular stent grafts depicted in the illustrations are intended to be generic; any resemblance to existing brand-specific stents is not an endorsement.

Flex Introducer Sheath (W.L. Gore \& Associates, Inc., Flagstaff, AZ, USA) over the Extra-Stiff wire; for stent grafts that do not require an introducer, we use a bareback technique with a GORE DrySeal Flex Introducer Sheath one size smaller than the outer stent graft diameter to advance the graft over the Extra-Stiff guidewire. (For example, if the stent graft required an $18 \mathrm{~F}$ sheath, we would use a $16 \mathrm{~F}$ introducer sheath to plug the femoral vessel before final percutaneous closure). After loading the selected stent graft device over the Extra-Stiff wire, we advance it under fluoroscopy to the targeted landing zone previously marked on the screen (Figure 4). Before we deploy the stent graft, we reduce the systolic blood pressure to $\leq 100 \mathrm{mmHg}$ and aim for a heart rate $\leq 90 \mathrm{bpm}$.

The stent graft(s) is deployed under fluoroscopic guidance (Figure 5). It is vital to ensure that the proximal and distal ends of the stent are deployed within the true lumen; we routinely use IVUS for this purpose, as well as to verify sizing of the stent graft (initially determined by

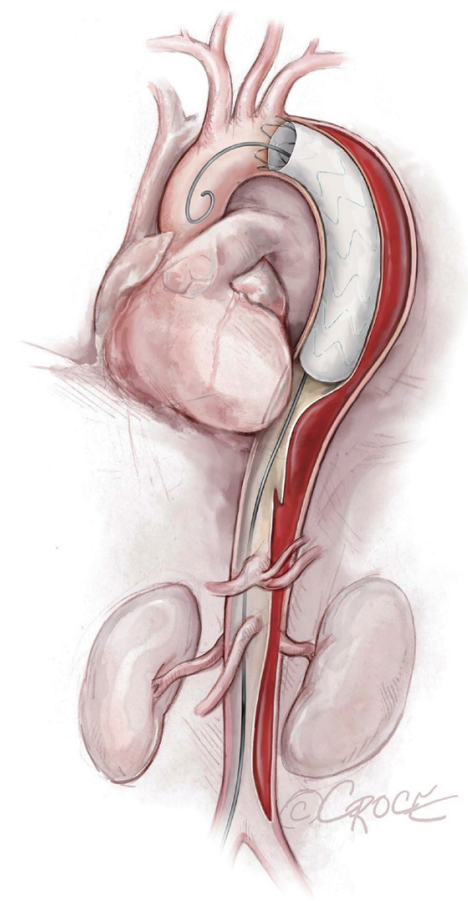

Figure 5 The first stent graft is deployed. The endovascular stent grafts depicted in the illustrations are intended to be generic; any resemblance to existing brand-specific stents is not an endorsement.

CT scan) and confirm adequate flow to the arteries after the stent graft has been placed. In addition, IVUS is important for assessing the ascending aorta, the status of the true and false lumen distal to the landed stent and the origins of the visceral vessels (from false lumen versus true lumen), as we prefer to extend the coverage of the descending thoracic aorta close to the celiac artery (Figure 6). If more than twothirds of the descending thoracic aorta has been covered, we insert a cerebrospinal drain whenever possible to decrease the risk for spinal cord ischemia. The drain generally can be removed within 24-72 hours, absent any concerning symptoms.

We typically use IVUS to examine the ascending aorta for retrograde aortic dissection. Unlike our approach with aortic aneurysms or chronic aortic dissections, in cases of acute distal dissection we avoid ballooning the stent graft to prevent retrograde ascending aortic dissection. In cases of compressed true lumen in the abdominal aorta or evidence of visceral or kidney malperfusion, we prefer to use the Zenith Dissection Endovascular Stent (Cook Medical), which includes the Zenith TX2 Dissection Endovascular 


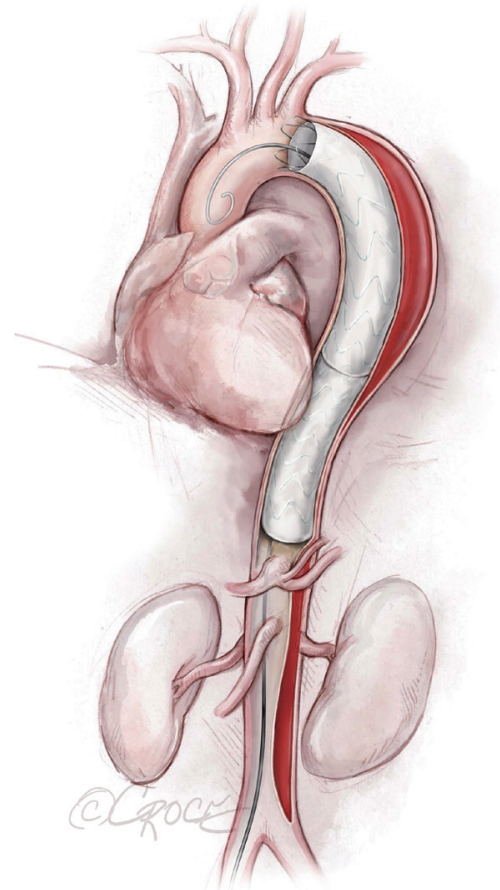

Figure 6 Completion TEVAR. Coverage of the descending thoracic aorta extends close to the celiac artery. The endovascular stent grafts depicted in the illustrations are intended to be generic; any resemblance to existing brand-specific stents is not an endorsement.

Graft with Pro-Form and Zenith Dissection Endovascular Stent (Figure 7).

\section{Completion and closure}

Once we have deployed the stent graft(s), we maintain mean blood pressure at 80-90 $\mathrm{mmHg}$ to reduce the risk for spinal cord ischemia; in cases of TBAD, we avoid very high systolic pressure (more than $140-150 \mathrm{mmHg}$ ) to preclude retrograde ascending aortic dissection, whereas in cases of thoracic aneurysm without dissection we are more aggressive, keeping the mean arterial pressure at $90-100 \mathrm{mmHg}$ and the systolic pressure at $150-160 \mathrm{mmHg}$ to avoid spinal cord ischemia.

If kidney function is acceptable, we use completion angiography to evaluate stent positioning in relationship to the head vessels and to the left subclavian artery, apposition after deployment and expansion of the true lumen of the thoracoabdominal aorta distal to the stent. When renal function is poor, IVUS can be used instead to limit the use of contrast, or else contrast material can be diluted

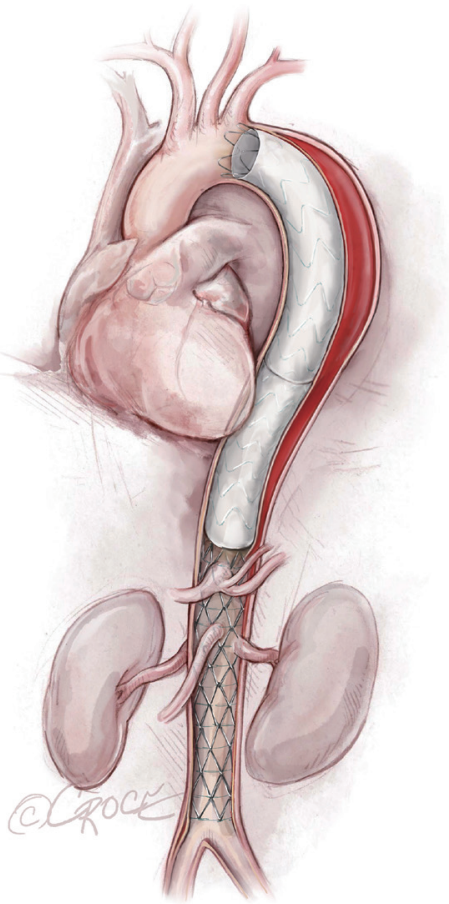

Figure 7 Zenith $^{\circledR}$ Dissection Endovascular Stent (Zenith TX2 Dissection Endovascular Graft) ends above the celiac axis and is placed across the visceral vessels and the renal arteries, extending to the aortic bifurcation.

(70\% contrast, $30 \%$ saline). The entire procedure can be performed with IVUS without intraoperative angiography, especially when renal insufficiency is present.

At the end of the procedure, the femoral sheath is removed and the incision is closed by using the two ProGlides placed initially. If a $14-20 \mathrm{~F}$ sheath was removed, we use an additional ProGlide if necessary, and if a 22-25 F sheath was removed, we use one to two additional ProGlides if necessary. The activated clotting time is reversed after the femoral sheath is removed.

\section{Additional considerations}

In our experience with TBAD, partial coverage of the left subclavian artery and more aggressive proximal landing may be required to prevent proximal type I endoleak; others also have advocated a more aggressive proximal landing zone (7). If there is concern about arm or spinal cord ischemia due to coverage of the left subclavian artery, we perform left subclavian-to-carotid bypass or transposition. Preoperative subclavian carotid bypass is necessary in certain cases, depending on the patient's anatomy and 
medical and surgical history (e.g., previous coronary artery bypass grafting with patent left internal mammary artery, left aortovenous fistula for hemodialysis, dominant left vertebral artery, symptomatic vertebrobasilar insufficiency or aneurysm extended to the left subclavian artery).

\section{Comments}

\section{Clinical results and advantages}

In a recent study on the endovascular treatment of complicated versus uncomplicated acute TBAD in 5,014 patients from the W.L Gore Global Registry, perioperative complication rates and thirty-day mortality were equally low for both groups, and midterm outcomes were positive (7). In another report from a prospective, nonrandomized dissection trial, 5-year outcomes for the Valiant Captivia endograft (Medtronic) included positive and sustained aortic remodeling (8). Results of the multicenter Zenith TX2 dissection endovascular graft study also were recently reported (9); in this study of 120 patients with complicated or uncomplicated type B aortic dissection, treated in the acute, subacute, or chronic phase, mortality was $6.6 \%$ in the acute cases with $4.2 \%$ permanent paraplegia and $2.5 \%$ stroke. No permanent paraplegia or permanent dialysis was reported in patients treated with concurrent placement of the Zenith Dissection Bare Stent. The retrograde type A dissection rate was low $(<1 \%)$, and morbidity and mortality rates were favorable. In our own experience treating 75 patients with acute TBAD, only one patient presented with a small ulceration proximal to the aortic stent and a small pseudoaneurysm proximal to the left subclavian artery three months after the index procedure (10). The caliber of that patient's ascending aorta was $4.5 \mathrm{~cm}$, which has been considered by others (11) to be a risk factor for retrograde dissection. To avoid retrograde ascending aortic dissection, the endovascular intervention should take place close to the subacute phase or within days of the initial presentation.

Some have shown worse outcomes if TEVAR is performed early after presentation $(12,13)$. In addition, for uncomplicated TBAD, a report based on data from the Vascular Quality Initiative TEVAR and complex endovascular aneurysm repair registry [2010-2019] indicates that endovascular repair within one to fourteen days of symptom onset is associated with a higher risk for reintervention within thirty days and within one year, although timing did not appear to predict mortality or other postoperative complications (14).

We recently reviewed the pros and cons of medical versus endovascular management for acute TBAD in an expert opinion article (15). We suggest that TEVAR for TBAD engenders favorable aortic remodeling, appears to be beneficial even in patients with high-risk clinical or radiographic attributes, and is procedurally low-risk when performed by experienced surgeons at comprehensive centers of aortic expertise. Recovery time is minimal, and patients can be discharged within 48 hours.

\section{Caveats}

Regarding the reported definitions for complicated and uncomplicated acute Type B aortic dissection, the 2014 European guidelines define complicated status as rupture, malperfusion, persistent or recurrent pain, refractory hypertension or early aortic expansion (16). According to the latest Society of Vascular Surgery and Society of Thoracic Surgeons reported standards, acute type B aortic dissection is considered complicated when there is rupture or malperfusion (2). The term uncomplicated is used when there is no rupture, malperfusion or high-risk features (2).

The length of the stent graft depends on pathological and anatomical considerations. Generally, for TBAD, we will cover from the proximal entry tear to a few centimeters above the origin of the celiac artery. We generally prefer to cover almost the entire length of the descending thoracic aorta, or at least two-thirds of the descending thoracic aorta in cases of acute TBAD, as more extensive coverage encourages thrombosis. However, in the presence of large intercostal arteries, we may foreshorten the distal landing zone to decrease the risk for paralysis. In cases of malperfusion with a collapsed true lumen, we have used the Zenith TX2 Dissection Endovascular Graft and the Zenith Dissection Bare stent (Cook Medical), a series of nitinol bare stents held together with monofilament sutures that is available in two diameters ( 36 and $46 \mathrm{~mm}$ ) and 3 lengths (80, 120 , and $180 \mathrm{~mm}$ ).

The caliber and quality of the landing zones and access vessels are crucial. Ideally, the proximal landing zone will consist of relatively normal, undissected aorta with a caliber of $4.2-4.5 \mathrm{~cm}$. The minimum landing zone length is $1.0 \mathrm{~cm}$. Additionally, a distance $>2.0 \mathrm{~cm}$ from the distal edge of the left carotid artery to the entry tear ensures an adequate seal of the aortic injury. When a stent deployment in zone 2 (17) is planned, with exclusion of the left subclavian artery, accessing the left common carotid artery through 
the left brachial artery and leaving a wire in place in case of inadvertent coverage of the left common carotid artery may be useful. A stent can then be quickly deployed in the left common carotid artery, protruding into the aorta, to push the stent graft away from the aortic wall and reestablish flow to the carotid artery.

An early report from the Arizona Heart Institute (18) emphasized the increased incidence of retrograde ascending aortic dissection in patients with acute type B aortic dissection versus descending thoracic aortic aneurysms. Avoiding a catastrophic retrograde dissection into the ascending aorta in acute TBAD requires a conservative approach at the proximal landing zone, avoidance of ballooning and oversizing of the stent graft not to exceed $10 \%$. In contrast, allowable oversizing at the distal landing zone is marginally more liberal, at $10-20 \%$.

We strongly believe that excessive wire manipulation plays an even more important role in producing adverse outcomes and provoking retrograde aortic dissection in patients with acute TBAD (10). Minimizing wire manipulation and knowing the appropriate wire and catheters to use are essential for achieving a highly successful outcome.

\section{Acknowledgments}

The authors thank Jeanie F. Woodruff, BS, ELS, of the Department of Scientific Publications at the Texas Heart Institute, for her editorial contributions, and Scott A. Weldon, MA, CMI, FAMI, of Baylor College of Medicine and Beth Croce, illustration editor at Annals of Cardiothoracic Surgery, for their artwork.

Funding: None.

\section{Footnote}

Conflicts of Interest: OP participates in clinical trials with and/or consults for Terumo Aortic and W.L. Gore \& Associates. ALH has no conflicts of interest to declare.

Open Access Statement: This is an Open Access article distributed in accordance with the Creative Commons Attribution-NonCommercial-NoDerivs 4.0 International License (CC BY-NC-ND 4.0), which permits the noncommercial replication and distribution of the article with the strict proviso that no changes or edits are made and the original work is properly cited (including links to both the formal publication through the relevant DOI and the license). See: https://creativecommons.org/licenses/by-nc-nd/4.0/.

\section{References}

1. Evangelista A, Isselbacher EM, Bossone E, et al. Insights From the International Registry of Acute Aortic Dissection: A 20-Year Experience of Collaborative Clinical Research. Circulation 2018;137:1846-60.

2. Lombardi JV, Hughes GC, Appoo JJ, et al. Society for Vascular Surgery (SVS) and Society of Thoracic Surgeons (STS) reporting standards for type B aortic dissections. J Vasc Surg 2020;71:723-47.

3. Tolenaar JL, Froehlich W, Jonker FH, et al. Predicting in-hospital mortality in acute type B aortic dissection: evidence from International Registry of Acute Aortic Dissection. Circulation 2014;130:S45-50.

4. Brunkwall J, Kasprzak P, Verhoeven E, et al. Endovascular repair of acute uncomplicated aortic type $\mathrm{B}$ dissection promotes aortic remodelling: 1 year results of the ADSORB trial. Eur J Vasc Endovasc Surg 2014;48:285-91.

5. Kamman AV, Brunkwall J, Verhoeven EL, et al. Predictors of aortic growth in uncomplicated type B aortic dissection from the Acute Dissection Stent Grafting or Best Medical Treatment (ADSORB) database. J Vasc Surg 2017;65:964971.e3.

6. Uchida T, Sadahiro M. Thoracic Endovascular Aortic Repair for Acute Aortic Dissection. Ann Vasc Dis 2018;11:464-72.

7. Spinelli D, Weaver FA, Azizzadeh A, et al. Endovascular treatment of complicated versus uncomplicated acute type B aortic dissection. J Thorac Cardiovasc Surg 2021. [Epub ahead of print].

8. Bavaria JE, Brinkman WT, Hughes GC, et al. Five-year outcomes of endovascular repair of complicated acute type B aortic dissections. J Thorac Cardiovasc Surg 2020. [Epub ahead of print].

9. Patel JJ, Kasprzak P, Pfister K, et al. Early outcomes associated with use of the Zenith TX2 Dissection Endovascular Graft for the treatment of Stanford type B aortic dissection. J Vasc Surg 2021;74:547-55.

10. Preventza O, Garcia A, Moeller K, et al. Retrograde Ascending Aortic Dissection After Thoracic Endovascular Aortic Repair for Distal Aortic Dissection or With Zone 0 Landing: Association, Risk Factors, and True Incidence. Ann Thorac Surg 2015;100:509-15.

11. Williams JB, Andersen ND, Bhattacharya SD, et al. 
Retrograde ascending aortic dissection as an early complication of thoracic endovascular aortic repair. J Vasc Surg 2012;55:1255-62.

12. Miyairi T, Miyata H, Chiba K, et al. Influence of Timing After Thoracic Endovascular Aortic Repair for Acute Type B Aortic Dissection. Ann Thorac Surg 2018;105:1392-6.

13. Desai ND, Gottret JP, Szeto WY, et al. Impact of timing on major complications after thoracic endovascular aortic repair for acute type B aortic dissection. J Thorac Cardiovasc Surg 2015;149:S151-6.

14. Torrent DJ, McFarland GE, Wang G, et al. Timing of thoracic endovascular aortic repair for uncomplicated acute type $\mathrm{B}$ aortic dissection and the association with complications. J Vasc Surg 2021;73:826-35.

15. Hong JC, Le Huu A, Preventza O. Medical or endovascular management of acute type $\mathrm{B}$ aortic

Cite this article as: Le Huu A, Preventza O. Endovascular repair of acute type B thoracic aortic dissection. Ann Cardiothorac Surg 2021;10(6):793-800. doi: 10.21037/acs2021-taes-26 dissection. J Thorac Cardiovasc Surg 2021. [Epub ahead of print].

16. Erbel R, Aboyans V, Boileau C, et al. 2014 ESC Guidelines on the diagnosis and treatment of aortic diseases:

Document covering acute and chronic aortic diseases of the thoracic and abdominal aorta of the adult. The Task Force for the Diagnosis and Treatment of Aortic Diseases of the European Society of Cardiology (ESC). Eur Heart J 2014;35:2873-926.

17. Ishimaru S. Endografting of the aortic arch. J Endovasc Ther 2004;11 Suppl 2:II62-71.

18. Kpodonu J, Preventza O, Ramaiah VG, et al. Retrograde type A dissection after endovascular stenting of the descending thoracic aorta. Is the risk real? Eur J Cardiothorac Surg 2008;33:1014-8. 